\section{Ectopic transgene expression in butterfly imaginal wing discs using vaccinia virus}

\author{
David L. Lewis ${ }^{1}$ and Craig R. Brunetti ${ }^{2}$ \\ ${ }^{1}$ Mirus Bio Corporation, Madison, WI, USA and ${ }^{2}$ Trent University, Peterborough, \\ ON, Canada
}

BioTechniques 40:48-54 (January 2006)

doi 10.2144/000112109

The rich diversity of wing color patterns within and between butterfly species makes butterflies an attractive system for studying the development and evolution of pattern formation. Study of the molecular and genetic basis of pattern formation in butterflies has revealed that a number of developmentally important genes possess expression patterns in the developing wing that correlate with the wing color patterns of the adult. For example, genes including engrailed/ invected, distal-less, cubitus interruptis, hedgehog, Notch, and spalt have expression patterns in the fifth larval instar and pupal wing imaginal discs that correlate with the formation of the eyespot, a color pattern composed of a series of concentric rings (1-5). Although these data suggest that these genes play a role in eyespot formation, the evidence is indirect. One way to directly determine the role that these and other genes play would be to ectopically express genes in the developing wings.

Currently, there are two viral vectors that enable transgene expression in vivo in arthropods, Sindbis virus (6) and baculovirus (7). In addition, transposonbased technology has also achieved ectopic expression in butterflies (8). Because baculoviruses naturally infect and can be potentially lethal to butterflies, their utility as a gene expression tool is limited. Furthermore, although Sindbis virus has been used as a gene expression vector in butterflies (6), this is an RNA virus and thus possesses a high mutation rate that could possibly impact expression of the gene of interest. In an effort to create an alternative viral system for ectopically expressing transgenes in butterfly pupal and larval wing tissue, we examined the use of vaccinia virus.

It has been reported that vaccinia virus, a vertebrate poxvirus, is able to infect gypsy moth cell lines in vitro (9). The vaccinia virus enters the gypsy moth cells and carries out both early and late viral protein synthesis as well as viral DNA replication (9). However, processing of viral late proteins does not occur, and therefore infectious virions are not produced. In this study, we set out to determine whether vaccinia virus could be used as a gene expression vector to ectopically express transgenes on the wings of butterflies in vivo.

To test this, we subcutaneously injected fifth instar Junonia coenia larva with vaccinia virus designed to express enhanced green fluorescent protein (EGFP) (10) through the larval cuticle in the area of the wing disc with 1-5 $\mu \mathrm{L}$ [approximately $10^{5}$ plaque forming units (pfus)] vaccinia virus EGFP diluted in Dulbecco's minimal essential medium along with $1 \%$ fetal bovine serum. The injected larva was maintained at $28^{\circ} \mathrm{C}$. Either fifth instar larval or 24-h pupal wing tissue were isolated, stained for $7 \mathrm{~min}$ with $100 \mathrm{nM}$ nuclear marker ToPro-3 (blue; Invitrogen, Carlsbad, CA, USA), and then examined for GFP expression (green) using a Model MRC600 laser-scanning confocal microscope (Bio-Rad Laboratories, Hercules, CA, USA). There was dramatic GFP expression in pupal stage butterfly wing tissue (Figure 1). Therefore, vaccinia virus can be used to ectopically express genes in $J$. coenia pupal stage wing tissue. However, we were unable to detect GFP expression in fifth instar stage wing discs (data not shown). Similar results were obtained with Sindbis virus, which was able to infect pupal, but not larval, wing discs of subcutaneously injected insects (Reference 6 and data not shown).

Although the ability to infect pupal stage wing tissue would allow the study of late stage patterning processes, such as induction of specific pigmentation genes, experimental evidence has shown that the majority of the prepatterning events that ultimately lead to the wing color pattern occur prior to the pupal stage (11). Thus study of prepatterning events requires ectopic expression during the prepupal stage of wing development. The data described above indicates that simply injecting virus into the larval hemolymph will not result in infection and transgene expression in the larval wing disc. It is possible that a physical barrier prevents infection of wing disc during this developmental stage. Viral particles likely infect pupal wing tissue by migrating through the wing tracheal system. Because the tracheal system is not fully developed in the larval stage wing discs (11), virus would not be able to use the tracheal system for dissemination to this tissue.

To circumvent the problem of viral accessibility to the wing tissue, we modified surgical techniques that had been used to manipulate butterfly wings either through ablation $(1,12-14)$ or wing disk transplantation (15). However, instead of physically removing the wing discs or damaging the wing, we used the surgical technique

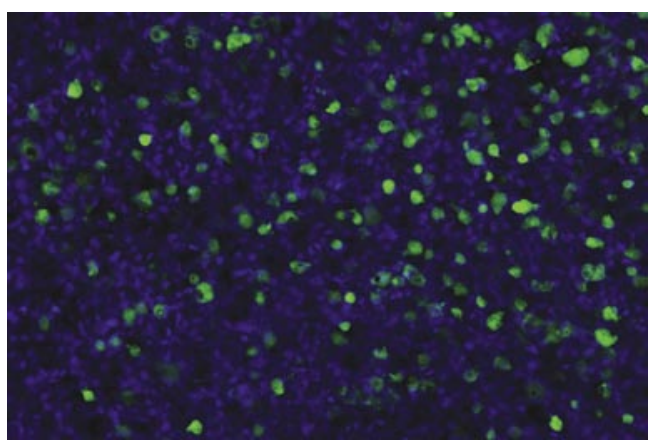

Figure 1. Vaccinia virus enhanced green fluorescent protein (EGFP) can infect and ectopically express a transgene in Junonia pupal wing tissue. Vaccinia virus-infected cells are shown in green (GFP), while cell nuclei are shown in blue (ToPro-3) using a MRC600 laserscanning confocal microscope. 
to expose the larval wing disc tissue so that it could be directly injected with viral particles. The technique involved anesthetizing fifth instar J. coenia larva by immersion in cold water for $10 \mathrm{~min}$. The larva were removed from the water and covered with phosphate-buffered saline (PBS) in a dissecting tray. A small incision was made along the midline of either the second (forewing) or third (hindwing) thoracic segment. Using tweezers, the wing disc was partially exteriorized, being sure not to damage the connecting tissue or trachea of the wing disc (Figure 2A). The wing discs were injected with approximately $10 \mathrm{~nL}$ (approximately 100 pfus) of vaccinia virus EGFP in Dulbecco's minimal essential medium along with $1 \%$ fetal bovine serum. The injection was done with a pulled glass needle (Figure 2B, yellow arrow) attached to a micromanipulator and microinjector set-up (Narishige, East Meadow, NY,
USA). After the needle was removed, the wing disc was carefully placed back inside of the body wall, and the hemolymph was allowed to melanize and close the surgical opening (Figure $2 \mathrm{C}$, yellow arrow). After $48 \mathrm{~h}$, the wing discs were removed, stained for 7 min with $100 \mathrm{nM}$ ToPro-3, and GFP expression was visualized using the MRC600 laser-scanning confocal microscope. This technique resulted in $100 \%$ survival of the larva, and over $75 \%$ of the surgically manipulated larva produced phenotypically normal adult wing tissue without signs of damage (data not shown). When larval wing discs were examined $48 \mathrm{~h}$ after direct injection, over $50 \%$ of the injected animals showed detectable GFP expression (Figure 2, D-F), demonstrating that viral infection of the wing discs had occurred. In addition, the GFP expression was localized to the site of injection, suggesting that this technique could be used to target gene expression to specific sites on the wing disc.

In this report, we have shown that vaccinia virus can be used to ectopically express a transgene in butterflies. The vaccinia viral vector and reporter gene used appeared to have no overt effect on the health of the insect or wing color pattern formation, an important property if vaccinia virus is to be used to express genes thought to be involved in this process. In addition, the fact that vaccinia virus, which is a vertebrate poxvirus, has the ability to infect butterflies in vivo as well as gypsy moth cells in vitro (9), suggests that vaccinia virus will also be a useful gene expression tool in other arthropod species for which classical genetic methods are either impractical or unavailable.

We have also described the development and use of direct injection
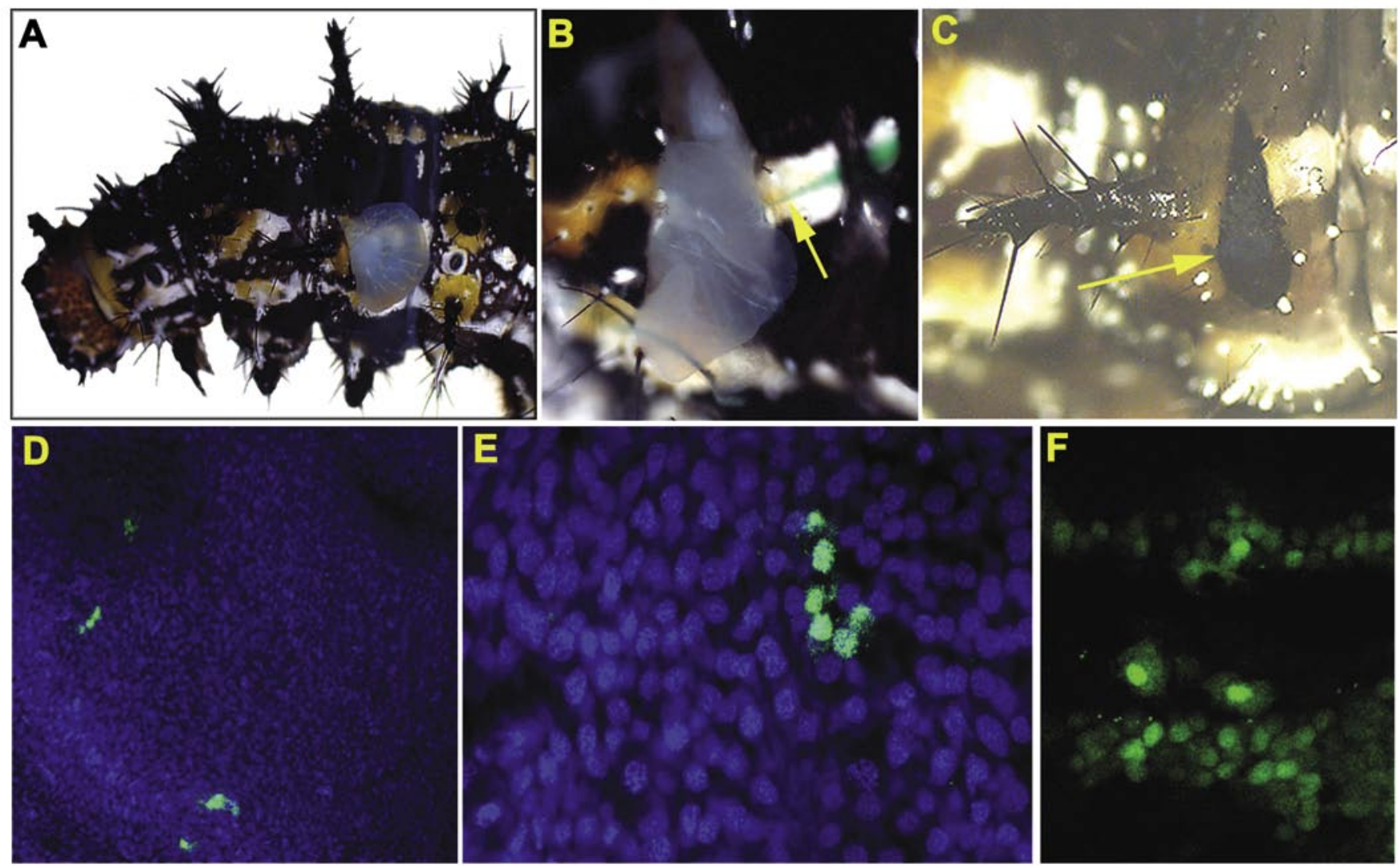

Figure 2. Ectopic expression of green fluorescent protein (GFP) upon direct injection of Junonia fifth instar larval wing discs. (A) The hindwing of a fifth instar Junonia coenia larva was partially exteriorized. (B) The wing disc was then injected with vaccinia virus enhanced GFP (EGFP) with a pulled glass needle (yellow arrow). (C) The wing disc was placed back inside of the body wall, and the hemolymph was allowed to melanize and close the surgical opening (yellow arrow). (D-F) After $48 \mathrm{~h}$, the wing discs were removed, and GFP (green) was visualized using a MRC600 laser-scanning confocal microscope. (D and E) Cell nuclei were visualized with ToPro-3 (blue). 
techniques as a means of vector delivery to larval wing imaginal discs. Because vaccinia virus does not productively infect insect cells (9), and thus the infections will be centered around the initial site of inoculation only, the ability to inject specific sites of the larval wing disc will allow us to directly target gene expression to particular areas of the wing at specific developmental times. This will enable us to further define the roles that a specific gene plays during color pattern formation. In addition, the development of surgical techniques to manipulate butterfly wing discs can be used for a variety of other experimental approaches to study pattern formation. For example, this method could be used to directly deliver antisense molecules such as morpholinos (16), as well as small interfering RNA (siRNA), expression plasmid constructs, or even purified proteins thought to be involved in color pattern formation such as decapentaplegic, wingless, or hedgehog. Together, the use of vaccinia viral-mediated transgene delivery and the direct injection techniques described here will make possible a wide range of in vivo manipulations to determine the molecular and genetic basis of butterfly wing color pattern development.

\section{ACKNOWLEDGMENTS}

We thank Sean Carroll for his advice and support and in whose laboratory this work was performed.

\section{COMPETING INTERESTS STATEMENT}

The authors declare no competing interests.

\section{REFERENCES}

1. Brakefield, P.M., J. Gates, D. Keys, F. Kesbeke, P. Wijngaarden, A. Monteiro, V. French, and S.B. Carroll. 1996. Development, plasticity and evolution of butterfly eyespot patterns. Nature 384:236-242.

2. Brunetti, C.R., J.E. Selegue, A. Monteiro, V. French, P.M. Brakefield, and S.B. Carroll. 2001. The generation and diversification of butterfly eyespot color patterns. Curr. Biol. 11:1578-1585.
3. Carroll, S.B., J. Gates, D.N. Keys, S.W. Paddock, G.E. Panganiban, J.E. Selegue, and J.A. Williams. 1994. Pattern formation and eyespot determination in butterfly wings. Science 265:109-114.

4. Keys, D.N., D.L. Lewis, J.E. Selegue, B.J. Pearson, L.V. Goodrich, R.L. Johnson, J. Gates, M.P. Scott, and S.B. Carroll. 1999. Recruitment of a hedgehog regulatory circuit in butterfly eyespot evolution. Science 283:532-534.

5. Reed, R.D. and M.S. Serfas. 2004. Butterfly wing pattern evolution is associated with changes in a Notch/Distal-less temporal pattern formation process. Curr. Biol. 14:11591166.

6.Lewis, D.L., M.A. DeCamillis, C.R. Brunetti, G. Halder, V.A. Kassner, J.E. Selegue, S. Higgs, and S.B. Carroll. 1999. Ectopic gene expression and homeotic transformations in arthropods using recombinant Sindbis viruses. Curr. Biol. 9:1279-1287.

7. Oppenheimer, D.I., A.M. MacNicol, and N.H. Patel. 1999. Functional conservation of the wingless-engrailed interaction as shown by a widely applicable baculovirus misexpression system. Curr. Biol. 9:1288-1296.

8.Marcus, J.M., D.M. Ramos, and A. Monteiro. 2004. Transformation of the butterfly Bicyclus anynana. Proc. R. Soc. B. Biol. Lett. 27(Suppl.):263-265.

9. Li, Y., S. Yuan, and R.W. Moyer. 1998. The non-permissive infection of insect (gypsy moth) LD-652 cells by vaccinia virus. Virology 248:74-82.

10.Wu, G.-Y., D.-J. Zou, T. Koothan, and H.T. Cline. 1995. Infection of frog neurons with vaccinia virus permits in vivo expression of foreign proteins. Neuron 14:681-684.

11.Nijhout, H.F. 1991. The Development and Evolution of Butterfly Wing Patterns. Smithsonian Institution Press, Washington, DC.

12.Hojyo, T. and H. Fujiwara. 1997. Reciprocal transplantation of wing discs between a wing deficient mutant (fl) and wild type of the silkworm, Bombyx mori. Dev. Growth Differ. 39:599-606.

13.Brakefield, P.M. and V. French. 1995. Eyespot development on butterfly wings: the epidermal response to damage. Dev. Biol. 168:98-111.

14.French, V. and P.M. Brakefield. 1995. Eyespot development on butterfly wings: the focal signal. Dev. Biol. 168:112-123.

15.Nijhout, H.F. 1980. Pattern formation on lepidopteran wings: determination of an eyespot. Dev. Biol. 80:267-274.

16.Angerer, L.M. and R.C. Angerer. 2004. Disruption of gene function using antisense morpholinos. Methods Cell Biol. 74:699-711.

Received 7 October 2005; accepted 21 November 2005.

Address correspondence to Craig $R$. Brunetti, Trent University, Department of Biology, 1600 East Bank Dr., Peterborough, ON K9J 7B8, Canada. e-mail: craigbrunetti@trentu.ca
To purchase reprints

of this article, contact

Reprints@BioTechniques.com 